\title{
Streamlines simulation of barrier fracture as a novel water shutoff technique
}

\author{
Abdullah Al-Najem • E. Pirayesh • M. Y. Soliman • \\ S. Siddiqui
}

Received: 7 March 2013/Accepted: 1 July 2013/Published online: 23 July 2013

(c) The Author(s) 2013. This article is published with open access at Springerlink.com

\begin{abstract}
Excessive water production has been a problem in the oil industry for many years. To handle this problem, many research projects have focused on developing conformance control systems. Conformance fracturing, a combination of hydraulic fracturing and water control, has proven to be an effective conformance control technique. Hydraulic fracturing is now the technology of choice for increasing well productivity. The chemistry of relative permeability modifiers has also undergone extensive change; the most notable result of which has been to prolong the life of water control treatments using relative permeability modifier (RPM) polymers. The purpose of this study was to investigate the application of barrierfracturing using streamline simulation. Barrier-fracturing is a novel idea that involves modifying the flow profile and diverting the displacing fluid by placing a fracture with essentially zero permeability deep into the reservoir. There are many ways to create a zero permeability fracture, examples of which include injection of cement or a conformance fluid into the fracture. In our study, we created several streamline simulation models to show the fidelity and validity of this innovative idea. The streamline simulation models that are presented in this paper range from a simple homogeneous reservoir to a very heterogeneous reservoir. The effect of different barrier-fracture lengths on the reservoir performance was analyzed. We also built streamline models for conventional mechanical and chemical water shutoff techniques (e.g. re-completion and RPM) to compare them with the novel barrier-fracture water shutoff technique. The resulting saturation distribution maps from the longer barrier-fracture clearly show the
\end{abstract}

A. Al-Najem ( $\varangle) \cdot$ E. Pirayesh · M. Y. Soliman · S. Siddiqui Texas Tech University, Lubbock, TX, USA

e-mail: abdullah.al-najem@ttu.edu power of a barrier-fracture to modify flow profile and divert the displacing fluid in comparison to conventional water shutoff techniques. Barrier-fractures helped improve oil recovery by delaying water-breakthrough and eventually improving the volumetric sweep efficiency.

Keywords Streamlines simulation - Sweep efficiency Reservoir · Management · Water shutoff · Barrier fracturing

\section{Introduction}

Conformance control refers to any solution designed to enhance the injection/production profile of a well by controlling the production of unwanted fluid. Conformance treatments may involve mechanical or chemical approaches or a combination of the two. Mechanical control may involve the use of packers or Inflow Control Devices (ICD's). Chemical approaches may be divided into two broad groups. One involves injecting a sealant into the reservoir to fully stop unwanted fluid flow. The other involves injection of relative permeability modifier (RPM) polymers to significantly reduce the relative permeability to water, while keeping the relative permeability to oil fairly intact.

It is very crucial to define reservoir characteristics and also conformance treatment conditions that could lead to successful chemical treatments and also provide guidelines for the application to actual fields. These might include thief zone temperature, vertical to horizontal permeability ratio, injection concentration and slug size. Tsau et al. (1985) used a simulator to model chemical treatments (polymer gels) and identify reservoir properties that strongly influence a conformance treatment. They found that low level of cross flow helped vertical conformance

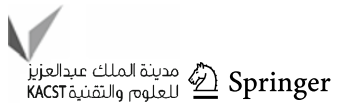


treatment and that a high ratio of permeability-thickness product between high and low permeability zones resulted in increased recovery. Melo and Aboud (2008) conducted over 100 conformance fracturing operations in Brazil, using conventional as well as lightweight proppants, and relative permeability modifiers. The authors presented a table of different treatments (design, logistics, materials,and equipment) versus obtained results (oil and water production over time), showing the improvements made over time. (Dang Cuong et al. 2011) history matched polymer gel behavior with experimental data and generated parameters for field scale simulation of the Lower Miocene reservoir of the White Tiger field. The field scale simulation results showed that implementing polymer gel treatment reduced excessive water production and improved oil recovery from unswept zones. Herbas et al. (2004) used a mechanistic field simulation to design water conformance treatments in Eastern Venezuelan HPHT reservoirs under different gel treatment scenarios. The simulation results showed water cut reduction from 90 to $30 \%$, matching trends observed in wells at different locations and gel treatment effects in typical Eastern Venezuelan reservoirs.

Another important design criterion that affects conformance treatments is the temperature near wellbore. So, to design the treatment based on a realistic temperatures rather than a bottom hole temperature, (Hardy et al. 1997) used temperature simulations to predict temperature near wellbore during water shutoff treatments and optimize treatment placement rates, fluid composition and shut-in times. Two North Sea field cases were presented. The first case illustrates how cooldown inside reservoir was used to place a treatment that would otherwise have gelled spontaneously at reservoir temperature. In the second case, the temperature simulations showed that several different activator compositions and concentrations were required for the early, intermediate and final treatment stages.

As we stated above, polymer flood works by reducing the mobility of water in highly conductive zones near the wellbore. However, injection of huge quantities of polymer near wellbore results in decreasing the drive fluid throughput and thus reservoir pressure support. To avoid such issues and also divert flow into lower permeability, unswept zones to mobilize bypassed oil, different conformance treatments have been proposed to form a block deep into the thief zone. Benson et al. (2007) proposed a novel deep penetrating mobility control method and used a numerical simulator to model the behavior of $\mathrm{pH}$-sensitive polymer in reactive porous media. The simulation results showed that $\mathrm{pH}$-sensitive polymer slug treatments improved vertical conformance in two layer radial and linear geometry floods. Another in-depth profile modification method was proposed by Garmeh et al. (2011) to use thermally activated polymer (TAP) which is an expandable submicron particulate of low viscosity and developed two simulation approaches to model properties of the thermally activated polymer (TAP) and its interaction with reservoir rock. Results showed that ultimate oil recovery and conformance control depend on thief zone temperature, vertical to horizontal permeability ratio, injection concentration and slug size, among other factors. Along the same line, Tobenna Okeke and Lane (2012) used a numerical simulator to model the potential effectiveness and performance of deep diverting gels (DDG) to plug thief zones deep within the reservoir by considering a wide range of reservoir characteristics and conditions. The authors compared the performance of the DDG to waterflooding and polymer flooding and found that a properly designed polymer flood had the highest NPV in all case comparisons, followed by DDG.

For such complex treatments, it is important to simulate the wellbore heat and pressure loss, reservoir temperature, polymer gelation and polymer adsorption. Unfortunately, these mechanisms have been modeled disparately by researchers. (Ansah et al. 2006) presented the first 3D, three phase, four component, pseudo-compositional, nonisothermal coupled reservoir/wellbore simulator that incorporates all these mechanisms through a rigorous tracking of all fluid concentrations during injection, shut-in and finally back flow of the fluids. Modeling the fourth phase, the conformance fluid, helps in tracking its location anywhere within the wellbore and reservoir during injection and flowback and thus maximizes the return and benefit of the various placements. The authors tested this methodology using coning and channeling examples in addition to two field treatments into two wells operated by Repsol YPF in Ecuador. The simulation results helped predict more accurate post-treatment water and hydrocarbon production and reduced operational and economic risks. Thornton et al. (2010) extended this work to optimize design and initialization of mechanical conformance using inflow control devices (ICD) and simulations helped in the optimum placement of ICD to minimize the water production. Also, Vasquez and Miranda (2010) used the same simulator presented by (Ansah et al. 2006) to evaluate performance of an RPM system under different scenarios and varying parameters. The simulation results showed that RPMs helped improve the injection profile by diverting water flow from high permeability into low permeability zones.

The purpose of this study was to investigate the application of the novel idea of barrier-fracturing using streamline simulation. We created several streamline simulation models to show the power of this innovative idea in modifying flow profile and delaying water breakthrough. The streamline simulation models range from a simple homogeneous reservoir to a heterogeneous reservoir with naturally fractured reservoirs (NFR). Following the introduction, we provide some background about streamline 
simulation technology and its demonstrated superiority, which made it the modeling tool of choice for us. Next, we discuss the proposed solution and the details of our simulation models. Simulation results and their interpretation are provided in the results section. Finally, our conclusions will be stated.

\section{Streamline simulation}

Streamlines are integrated curves that are locally tangential to a defined velocity field at a given instant in time (DattaGupta and King 2007 and Thiele et al. 2010) as illustrated in Fig. 1. Modeling fluid flow and transport using streamlines dates back to the study of well pattern and total recovery by Muskat and Wyckoff in 1934. A comprehensive historical overview of earlier streamlines work can be found in Batycky (1997); (Datta-Gupta and King 1998);

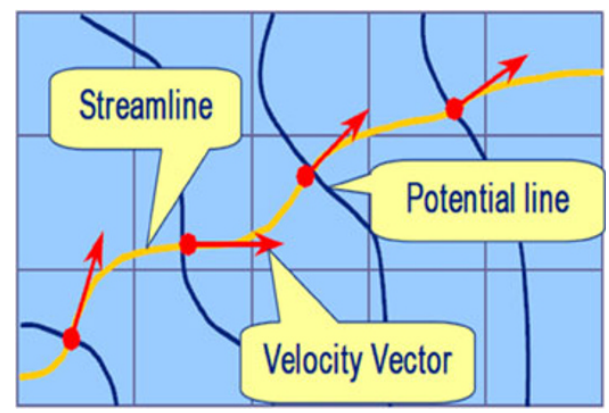

Fig. 1 Streamline tracing and velocity vector mapping in a saturation grid (Ibrahim et al. 2007)
(Thiele 2001); Moreno et al. (2004);and Datta-Gupta and King (2007).

Streamline simulation is an Implicit Pressure Explicit Saturation (IMPES) type reservoir simulation that solves the pressure equation implicitly and then solves the saturation/conservation equations explicitly. Thus, streamline simulators operate on the principle of decoupling the pressure equation from the saturation equation. This simplification allows a heterogeneous 3D domain to be decomposed into a number of 1D streamlines where all fluid calculations are carried out.

Figure 2 is a generalized flow diagram for streamline simulation. The 3D grid is first initialized starting with input data such as grid geometry, rock and fluid properties, well locations, injection rates and boundary conditions. Then, using finite difference (FD) approximations, the pressure distribution is derived to generate instantaneous velocity vectors perpendicular to the computed pressure contours. The velocity field is then used to trace the streamlines. The specified wells (i.e. source or sink) and boundary conditions govern the initiation and termination of all traced streamlines, which once established make up the second grid system needed to help solve the fluid flow equation along the streamlines. The second grid system works under a local time-step controlled by fluid movement computations, making it a time-variant grid. The solution obtained from the second grid is then mapped back onto the original 3D Cartesian grid to account for fluid phase distribution and saturations. For subsequent cycles, the pressure is solved again and the streamlines are redrawn. The process continues until the end of simulation.

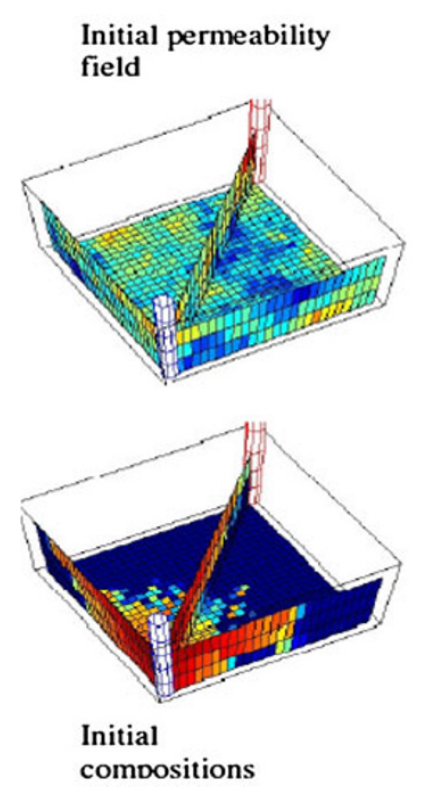

Initial permeability
1. Solve for pressure

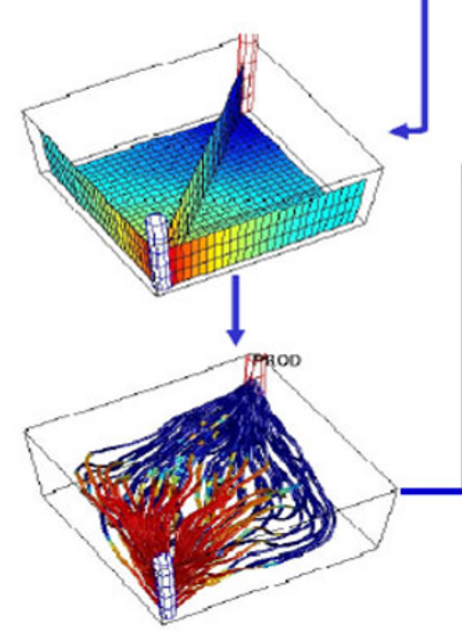

2. Find velocity field, trace streamlines

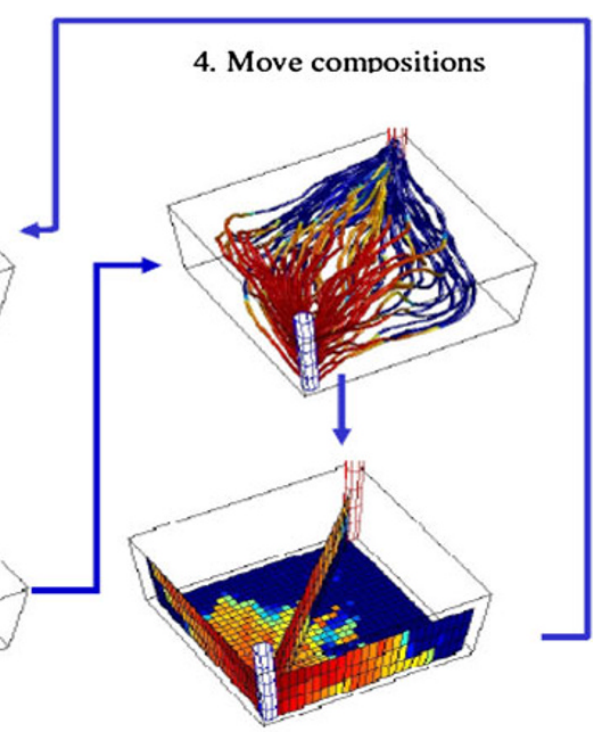

5. Map compositions to pressure grid, account for gravity, repeat

Fig. 2 Generalized flow diagram for streamline simulation (Gerritsen 2008)

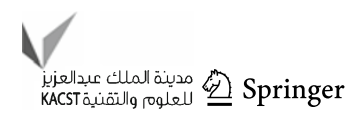


This dual grid approach distinguishes streamline simulators from conventional finite difference simulators.

This simulation process involves many different mathematical calculations on both grid systems to solve the pressure and transport equations. The following references thoroughly explain the mathematical formulations. These references include but are not limited to (Batycky 1997; Ingebrigtsen et al. 1999; Doi and Suzuki 2000; Lolomari et al. 2000; Gautier et al. 2001; Jessen and Orr Jr. 2002; Di Donato et al. 2003; Moreno et al. 2004; Gerritsen et al. 2005; Mallison et al. 2006; Cheng et al. 2006).

There are several advantages for employing streamline simulations for modeling fluid flow. One of the main

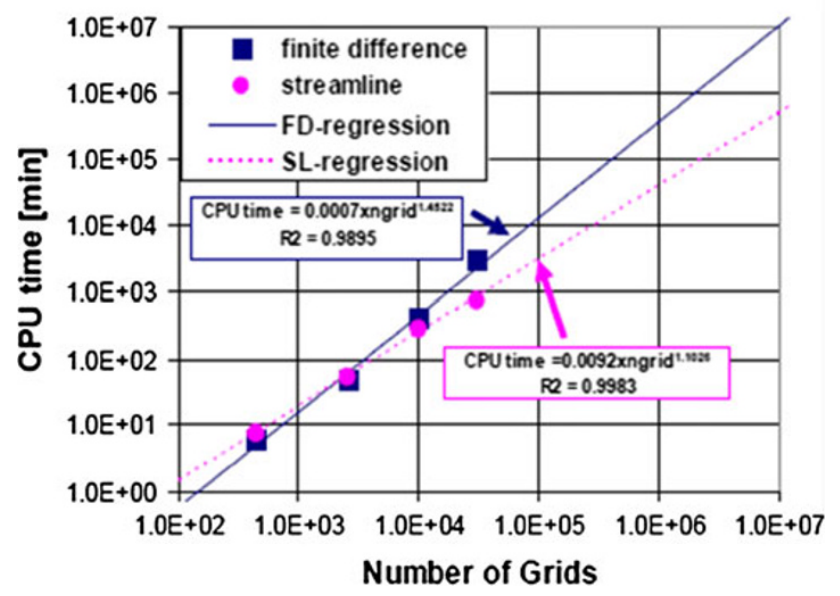

Fig. 3 Scaling of CPU time: streamlines vs. finite difference (Osako and Datta-Gupta 2007) advantages that attracted researchers to streamline technology in the first place was its computational speed. Due to their 1D nature, transport calculations are not constrained by grid instability, allowing for larger time steps and minimizing numerical diffusion. Batycky (1997) and Datta-Gupta and King (2007) reported that streamline technique exhibits a near-linear scaling of the CPU time and was faster than FD simulation by factors of 1-3 orders of magnitude especially for large models (i.e. $>10,000$ grid blocks) as can be seen from Fig. 3 .

Another advantage is the visualization potential offered by streamlines simulations. For example, visualizing the source-sink relationships based on streamline density can help obtain a quantitative flow indicator. This visualization is extremely useful in optimizing waterfloods/gas floods because the benefits of injection can be easily quantified over the time as can be seen in Fig. 4.

In addition, this powerful aspect of streamlines visualization, with the help of the quantitative flow indicators, can assist in identifying fluid loss to wells outside a pattern and then balancing different patterns using well allocation factors (WAF) between injectors and producers. Figure 5 shows a simple case of using streamlines information to help re-balancing of rates. To optimize injection efficiency of each injector, the rates are changed from one time to another till even distribution of streamlines associated with injectors or producers are obtained.

These are just some of the advantages of streamlines methods. We would highly recommend that the reader refers to AlNajem et al. (2012) for attaining a better idea about the different advantages of streamlines technology and also the
Fig. 4 Streamlines capturing sweep/drainage areas associated with injectors and producers (Thiele 2001)
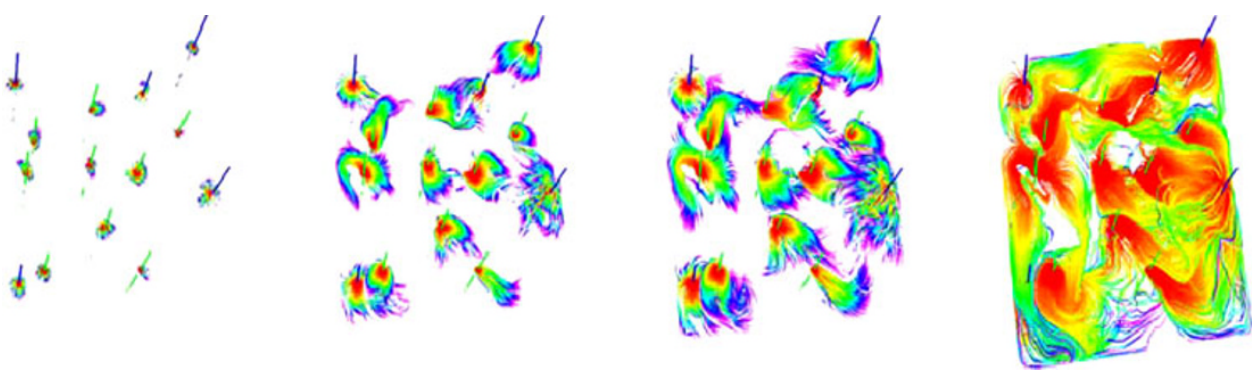

Fig. 5 Streamlines information aid in re-balancing rates in different patterns (Thiele 2001)
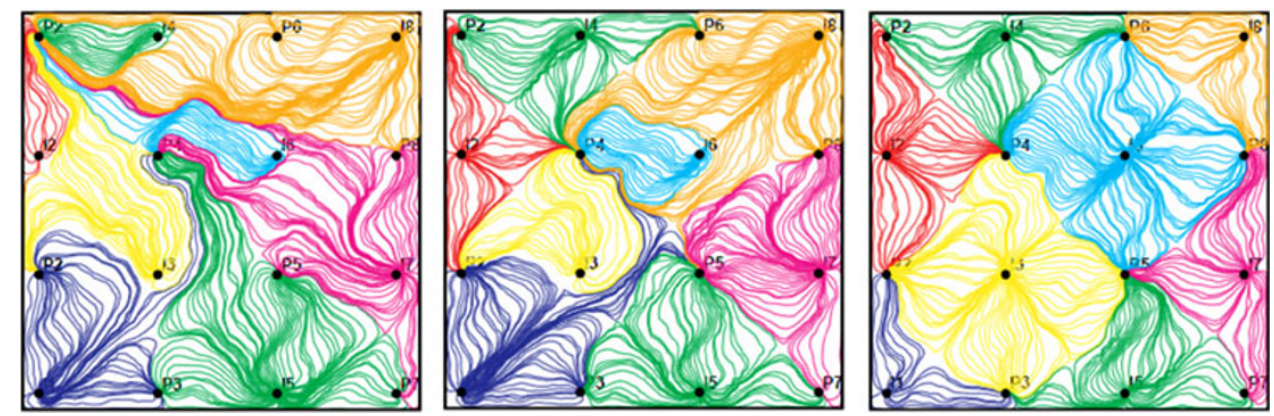
wide range of petroleum engineering applications that symbolize the relevance and validity of streamline simulation in addressing reservoir engineering concerns.

Like FD simulation, streamline simulation has its limitations. Two important ones are mapping between coordinates and modeling of fluid flow complex physics. Streamline simulation contains two separate grids, an underlying physical grid where the pressures and the velocities are calculated, and the streamline time-of-flight grid where the fluid transportation is calculated. Streamlines are re-generated at each pressure update. This means that the saturations from the old set of streamlines must be mapped back to a new set of streamlines. Streamlines transport saturations rather than conserved volumes, which are only implicitly defined in the time-of-flight coordinate. Because of the re-sampling of implicit volume from timeof-flight coordinates to physical coordinates, potential mass balance errors and to some extent, numerical dispersion may be introduced.

Another major limitation of streamline simulation results from its main advantage defined above: computational speed. When dealing with complex physics like high compressibility, capillary effects and phase behavior, the computational speed decreases. This is due to the need for more frequent re-sampling of streamlines, which means more frequent solving of the pressure equation.

Streamline-based flow simulation has made significant advances in the last 15 years. Today's simulators are fully three-dimensional and fully compressible and they account for gravity, fracture flow, and non-uniform conditions as well as complex well controls. Most recent advances also allow for compositional and thermal displacements.

\section{Description of the streamline simulation model}

One-fourth of five spot pattern streamline model was built using FRONTSIM, the Schlumberger streamline simulator. The base case 3D simulation model is 660 by 660 by $490 \mathrm{ft}$ with a Cartesian grid of $44 \times 41 \times 13$ grid blocks in the $\mathrm{x}, \mathrm{y}$ and $z$ directions, respectively. The sizes of each grid block in both $\mathrm{x}$ and $\mathrm{y}$ directions were designed using a geometric gridding coefficient of 1.2 ; the size of the layers in the $\mathrm{z}$ direction varies as shown in Fig. 4. We intentionally designed the grids in this manner to aid the placement and modeling of the barrier fracturing deep in the reservoir. Both injector and producer were completed in the first ten layers and the injection and production rates were maintained constant at 1,000 STB/D. Porosity is $24 \%$ and constant, and the vertical permeability is maintained at $10 \%$ of horizontal permeabilities (i.e. $\mathrm{K}_{x}$ and $\mathrm{K}_{y}$ ). The simulation was run for 6,000 days ( $>16$ years) and the barrier fracturing is modeled by reducing the grid's permeability in all directions to a very small number (10e-11 md). Figure 5 compares the oil saturation along the streamlines in the model at the breakthrough time and at the end of simulation (6,000 days) where, after placing the barrier fracture, the streamlines had to go around it to reach the producer, which would delay the breakthrough of water.

\section{Application of the model-numerical cases}

In this section, we present five numerical cases to show the use of the streamline simulation to model barrier fracturing. As mentioned earlier, these cases included the modeling of a simple homogeneous reservoir, a homogeneous reservoir with included areas of high permeability, low permeability, and a heterogeneous reservoir. By going with the conventional wisdom, we modeled the barrier fracture right after breakthrough occurs in all of the streamline models. This is done using the "restart" simulation files at the time of breakthrough to initialize the new streamline model where the barrier fracture has been included. We also built streamline models for the conventional mechanical and chemical water-shut off techniques such as re-completion and RPM, respectively, to compare them with the novel barrier fracture water shutoff concept.

Because this innovative concept revolves around modifying the flow profile deep in the reservoir, the
Fig. $61 / 4$ of five spot pattern streamline model with grids constructed using a geometric gridding coefficient of 1.2 in the $\mathrm{x}$ and $\mathrm{y}$ directions and variable layers' sizes in the $\mathrm{z}$ direction
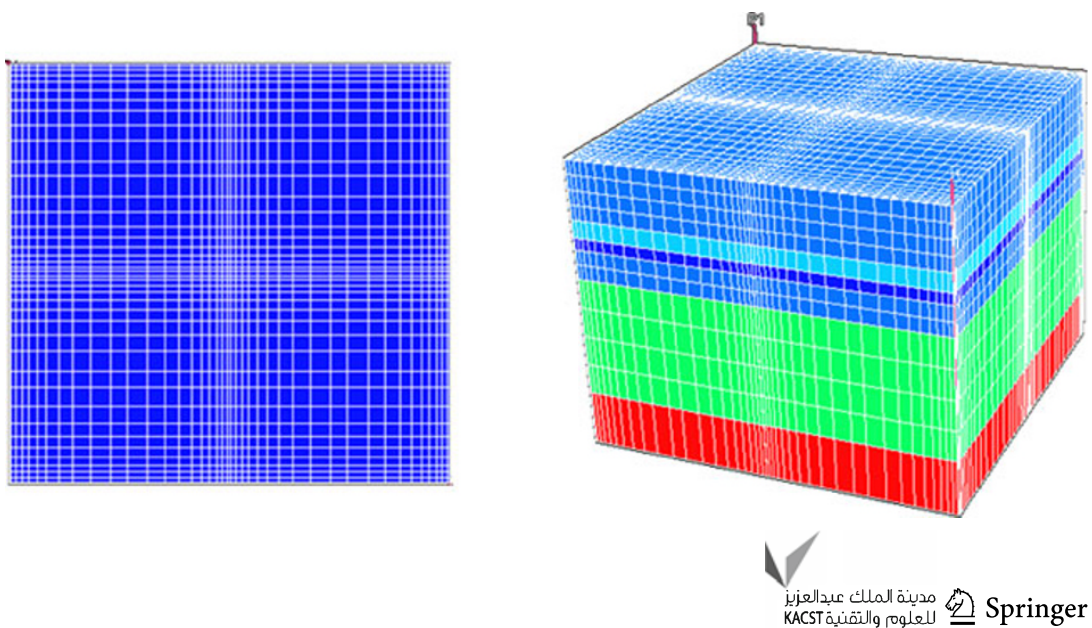
Fig. 7 Oil saturations along streamlines at time of BT and 6,000 days for "No_Frac" and "Barrier_Frac" cases showing no streamlines crossing the barrier fracture
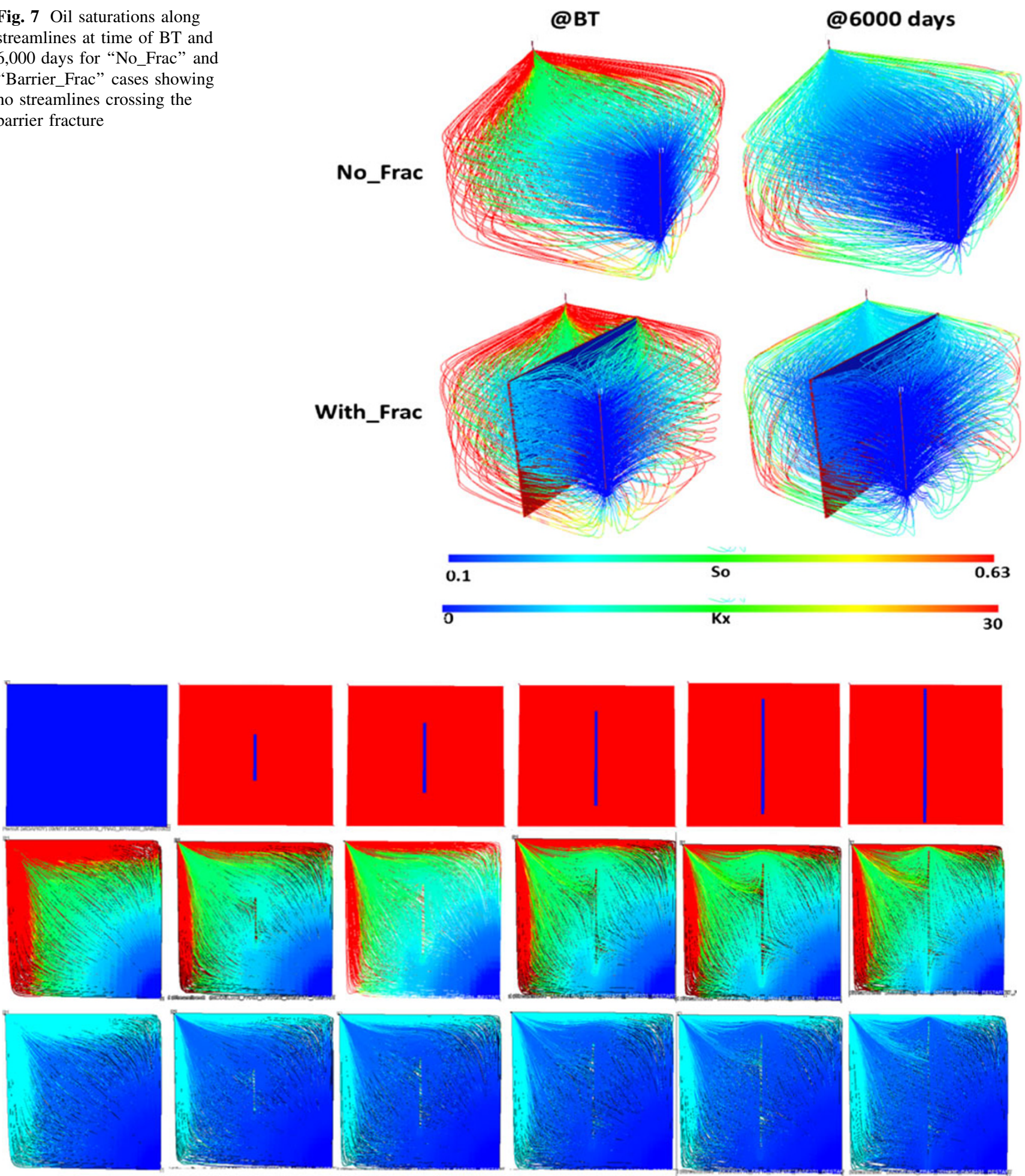

Fig. 8 Snapshots of oil and water saturations along the streamlines at time 2,190 days

placement of the barrier fracture is somewhat contrived. However, the effects of different barrier fracture lengths on reservoir performance and sweep efficiency were analyzed to find the optimum length that would serve the purpose of placing a barrier fracture in the reservoir.
This barrier fracture length sensitivity analysis was carried out only with the homogenous reservoir case and since this optimum length gave the maximum reservoir performance, we utilized the same length when modeling the other numerical cases. 


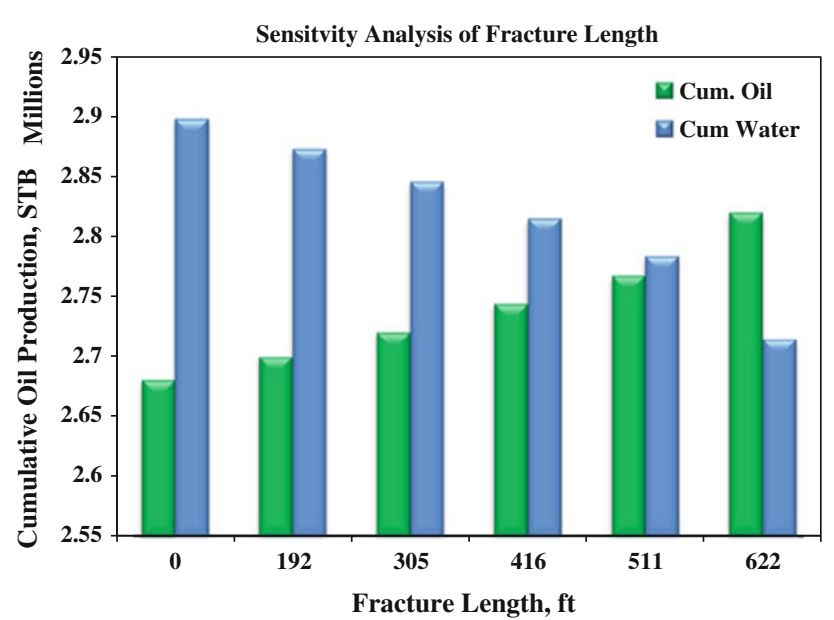

Fig. 9 Sensitivity analysis of fracture length showing increase in oil and decrease in water with increasing fracture length-Homogeneous reservoir

\section{Case-1: homogenous reservoir}

This is a simple homogenous and isotropic model with permeability in the $\mathrm{x}$ direction of $30 \mathrm{md}$ and permeability in the y direction of $45 \mathrm{md}$. As we stated before, the vertical permeability is everywhere maintained at $10 \%$ of the horizontal permeability. A base case of this model was run for the full simulation time to determine the breakthrough time and also to output the required "restart" files for use in the other cases including the barrier fracture. The barrier fracture was placed in the middle of the reservoir as shown by Fig. 6 and its impact should start right after the breakthrough time at 1,590 days.

In this model, we sensitized on the length of the barrier fracture. Five different barrier fracture lengths (192, 305, 416, 511, and $622 \mathrm{ft}$ ) were tried, as shown in Fig. 6. Figure 6 depicts snapshots of oil and water saturation along streamlines at time 2,190 days (i.e. 600 days after BT time) and also shows the different lengths of barrier fractures placed in the middle of the reservoir. It is very clear that injected water along the streamlines in the model with the longest barrier fracture $(622 \mathrm{ft})$ had to move along longer paths, which helped delay water movement toward the producer. Figure 7 shows the cumulative oil production and cumulative water production from the different streamline models with different barrier fracture lengths. We see here that the base case (no barrier fracture) produced the least cumulative oil recovery and the highest cumulative water production. In addition, based on the sensitivity analysis of barrier fracture length, we see that as the length increases, the oil production is increases and water production decreases. So, the optimum barrier fracture length is $622 \mathrm{ft}$, which gave the highest percentage increase in oil recovery as shown by Fig. 8 and lowest water production as shown by Fig. 9 .

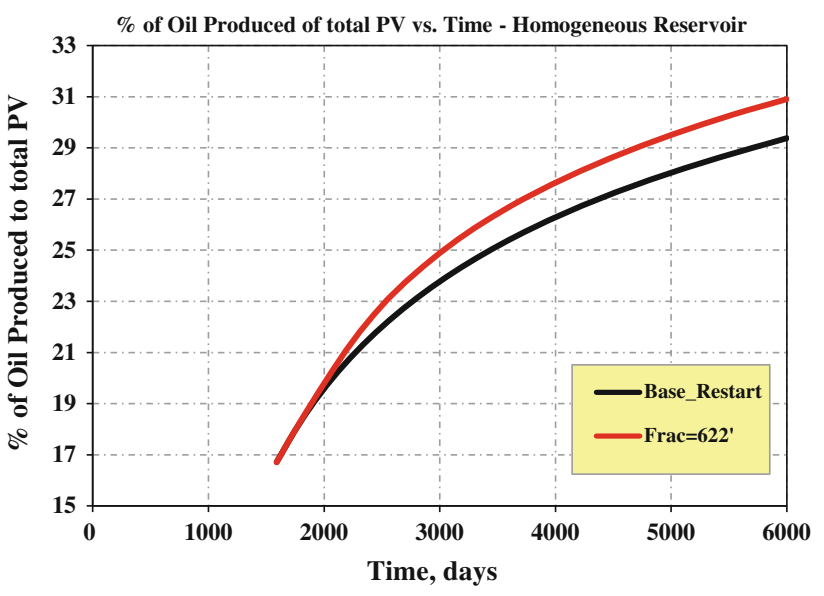

Fig. $10 \%$ of oil produced of total PV for the base and barrier fracture $622 \mathrm{ft}$ cases

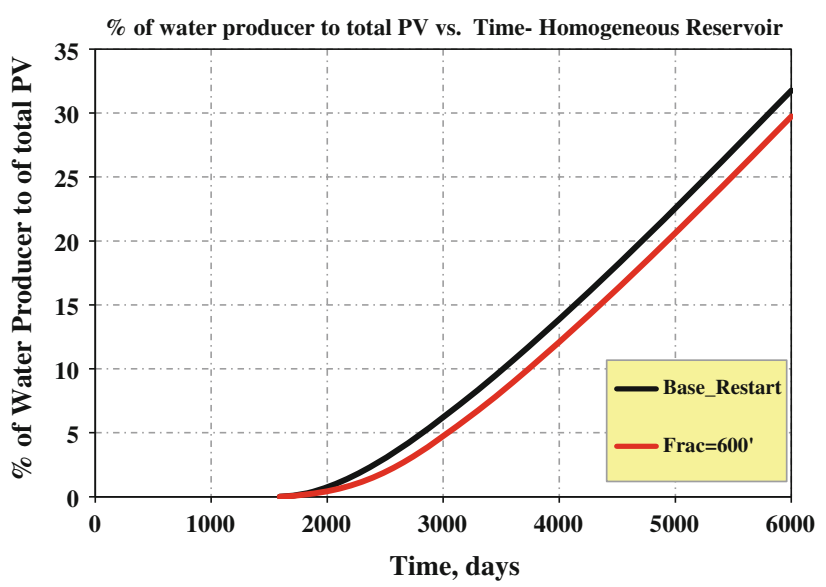

Fig. $11 \%$ of water produced of total PV for the base and barrier fracture $622 \mathrm{ft}$ cases

\section{Comparison of conventional water shutoff and barrier fracturing}

In practical field management, operators usually react to the production of water by either re-completing the well to stay away from water producing zones or by injecting relative permeability modifiers (RPM). So, because the "no-action" case that we presented might not be realistic, we built streamline models for the conventional mechanical and chemical water shutoff techniques (i.e. re-completion and RPM) to determine the superiority of the novel barrier fracture water shutoff technique.

The mechnical water shutoff modeling was basically done by identifying the water producing zones and keeping the completion far from the trouble zones. So, for this streamline model, the producer was re-completed in the first 5 layers. On the other hand, modeling the chemical water shutoff (i.e. RPM) was done by reducing the relative 

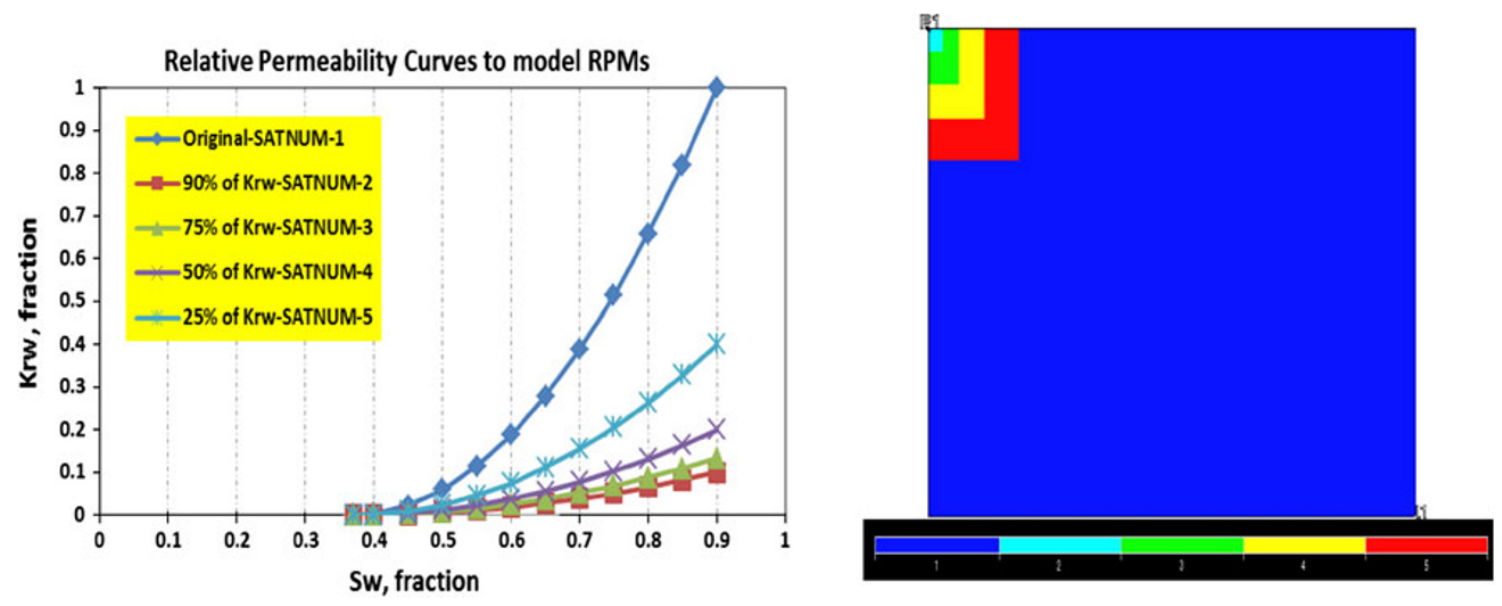

Fig. 12 RPM modeling using different saturations regions (SATNUM)

permeabilitiy to water around the area of the well. Again, to be practical, and since we know the RPM impact will be lower as it goes deeper into the reservoir, we designed this case with five different water relative permeability curves using different saturation regions. Near the well, the water relative permeability is reduced by $90 \%$ (i.e. SATNUM-2) and this percentage decreases to reach zero, which is represented by the original relative permeability curves (SATNUM-1) as shown in Fig. 10.

The results of comparing these different water shutoff techniques are depicted in Figs. 11 and 12. These figures show that the barrier-fracture technique produced the highest oil recovery. It was very interesting to observe that the RPM model was producing as much as the barrier-fracture model for more than 600 days, but with time it started to produce less. This tells us that the barrier fracture is a long-term solution to the water problem unlike chemical treatments, the effects of which will deteriorate with time and ultimately produce as little as the re-completion water shutoff technique. Even though re-completing the producer was a permanent remedial action, the barrier fracture placed deep in the reservoir gave much better results in terms of increasing oil recovery (oil increased by $>4.6 \%$ ) and reducing water production (water increased by $6 \%$ ).

\section{Case-2 and 3: Effects of high and low permeabilities}

In Cases 2 and 3, we further explored the applicability of this innovative concept with the presence of high and low permeabilities in the reservoir. Fig. 13a shows the distribution and magnitude of the low permeabilities in the reservoir in addition to the placement of the barrier fracture. The low permeabilities are only $1 \%$ (3md), $0.1 \%$ $(0.3 \mathrm{md})$ and zero for parts of the overall permeability in this $300 \mathrm{md}$ model. Figure 13b, c show the oil saturation along the streamlines at time 840 days, which is the time of

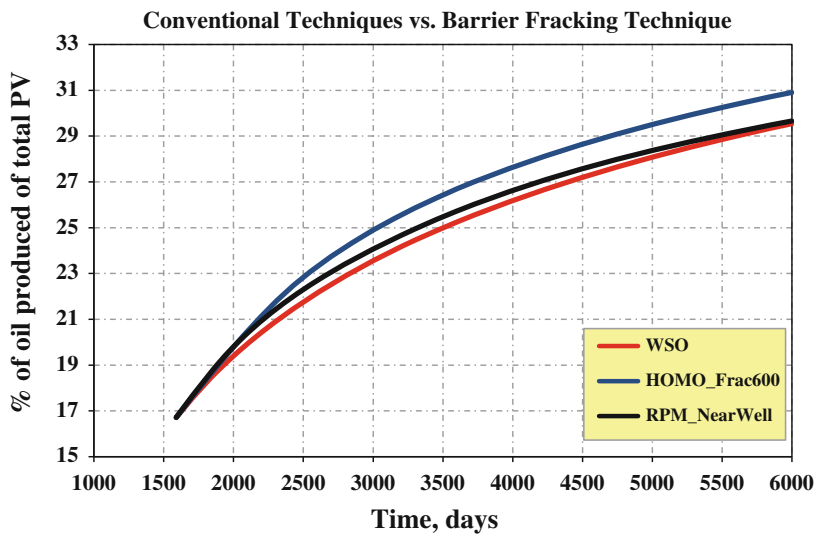

Fig. 13 Cumulative oil production comparison of conventional techniques vs. barrier fracture technique

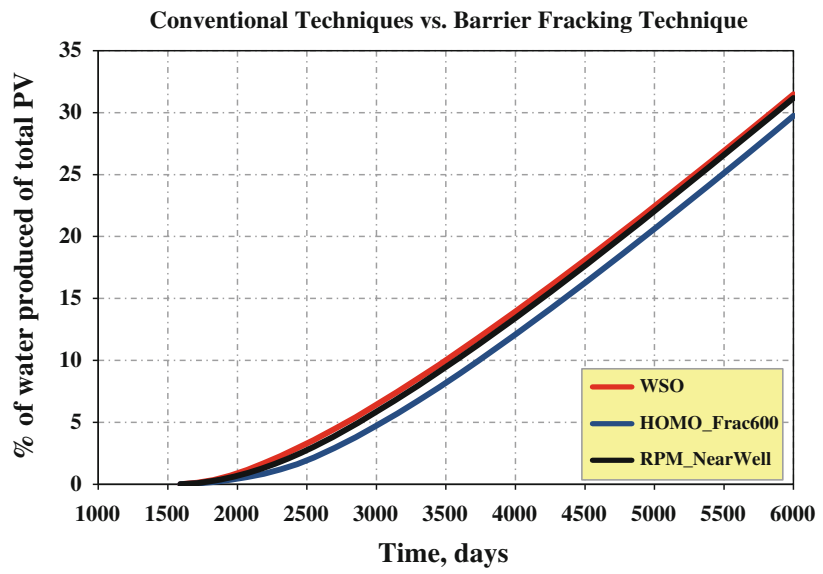

Fig. 14 Cumulative water production comparison of conventional techniques vs. barrier fracture technique

breakthrough, and at the end of simulation (6,000 days). We see here how streamlines are interacting with the low permeabilities and that no streamlines pass through them and that the barrier fracture is almost blocking any further movement of injected water. 
Fig. 15 Permeability distribution and oil saturations along streamlines for the low case

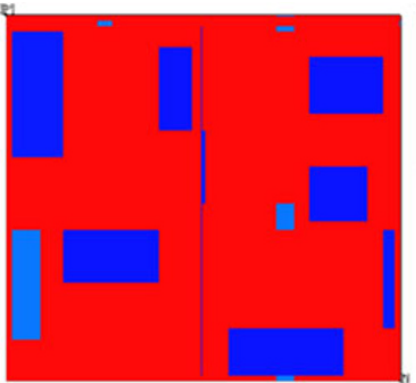

(a)

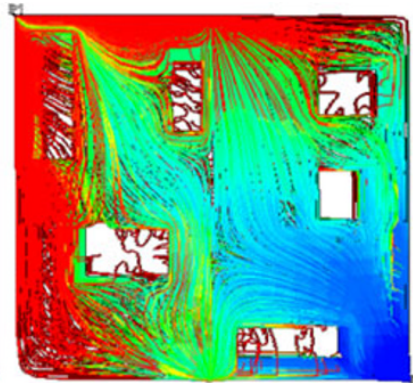

(b)

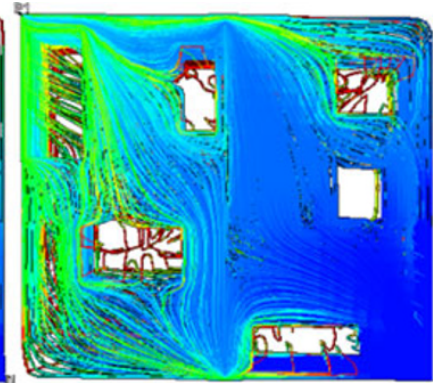

(c)
Fig. 16 Permeability distribution and oil saturations along streamlines for the high zones case

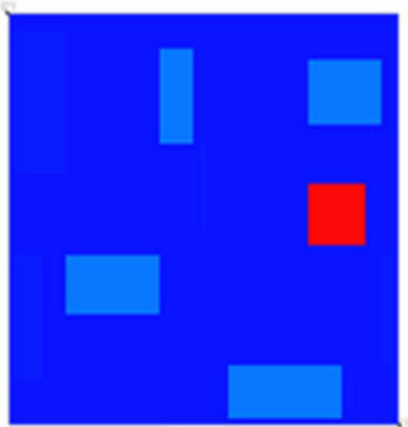

(a)

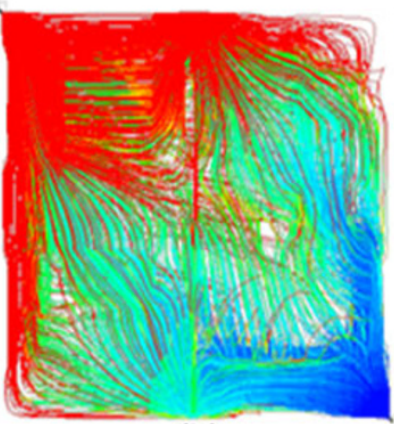

(b)

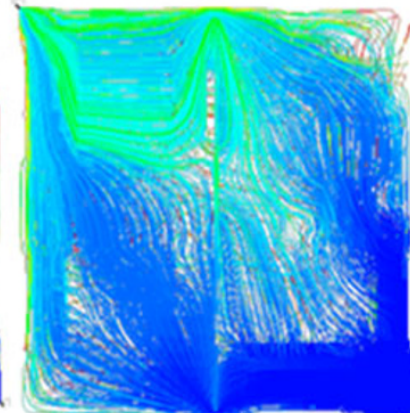

(c)
Similarly, Fig. 14a depicts the distribution and magnitude of the high permeabilities in the reservoir in addition to the placement of the barrier fracture. The high permeabilities are $1,000 \%(300,000 \mathrm{md})$ and $100 \%(30,000 \mathrm{md})$ of the overall permeability in this 300 md model. Fig. 14b, c show the oil saturation along the streamlines at time 750 days, which is the time of breakthrough, and at the end of simulation (6,000 days). We see here that most of the flow is coming through the high permeabilities, which are very clearly surrounded by higher water saturation values.

Figures 15 and 16 show the percentage of oil produced of total pore volume and percentage of water produced of total pore volume obtained for the low and high permeabilities. As we see here, in both cases (red vs. black and green vs. blue), the barrier fracture helped to improve oil recovery and reduce water production.

\section{Case-4: heterogeneous reservoir}

This is a synthetic heterogeneous and anisotropic model where the permeability was distributed log normally in all directions. Similar procedures as in the homogeneous model were followed to place the barrier fracture after the breakthrough of water and initialize using the "restart" files.

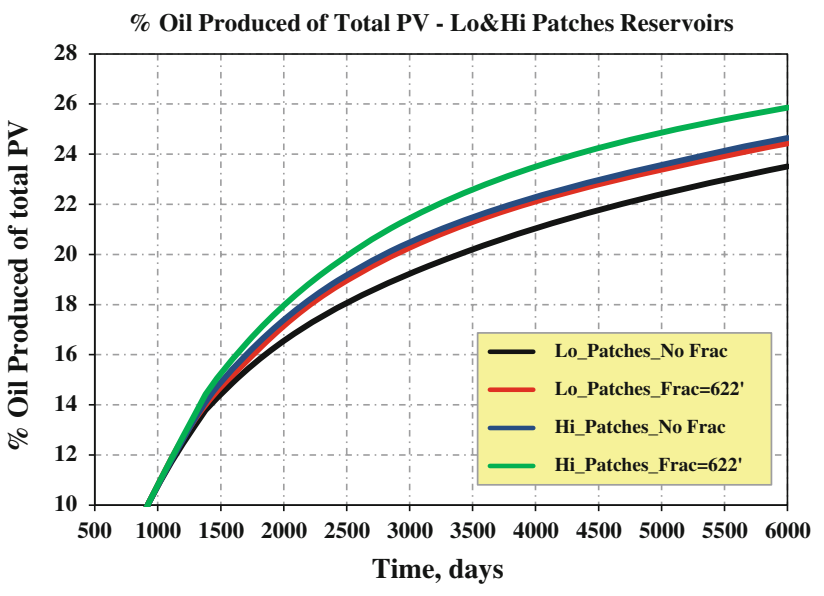

Fig. $17 \%$ oil produced of total PV for the Lo and high zones cases

Like the homogeneous model, in this model we sensitized on five different barrier-fracture lengths (192, 305, 416, 511, and $622 \mathrm{ft}$ ) as shown in Fig. 17. Figure 17 depicts snapshots of oil and water saturation along streamlines at time 2,190 days (i.e. 480 days after BT) and also illustrates the different lengths of barrier fractures placed in the middle of the reservoir. It is very clear that injected water along the streamlines in the model with the longest barrier fracture (622 ft) had to move along longer paths, which helped delay water movement toward the producer. Fig. 18 shows the 
cumulative oil production and cumulative water production obtained from the different streamlines with different barrier-fracture lengths. Like the homogeneous model, we see here that the base case (no barrier fracture) produced the least cumulative oil recovery and the highest cumulative water production. In addition, based on the sensitivity analysis of barrier-fracture length, we see that as the fracture length increases, the oil production increases as shown in Fig. 19 and water production decreases as in Fig. 20. So, even with the realistic heterogeneity distribution, Fig. 21 the barrierfracture concept is helping to improve Fig. 22 ultimate recovery and sweep efficiency.

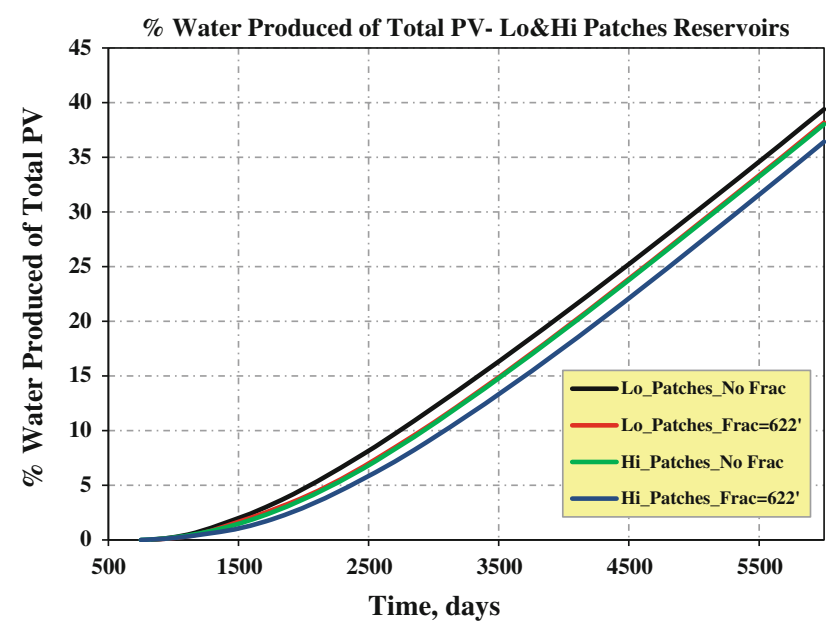

Fig. $18 \%$ water produced of total PV for the Lo and high zones
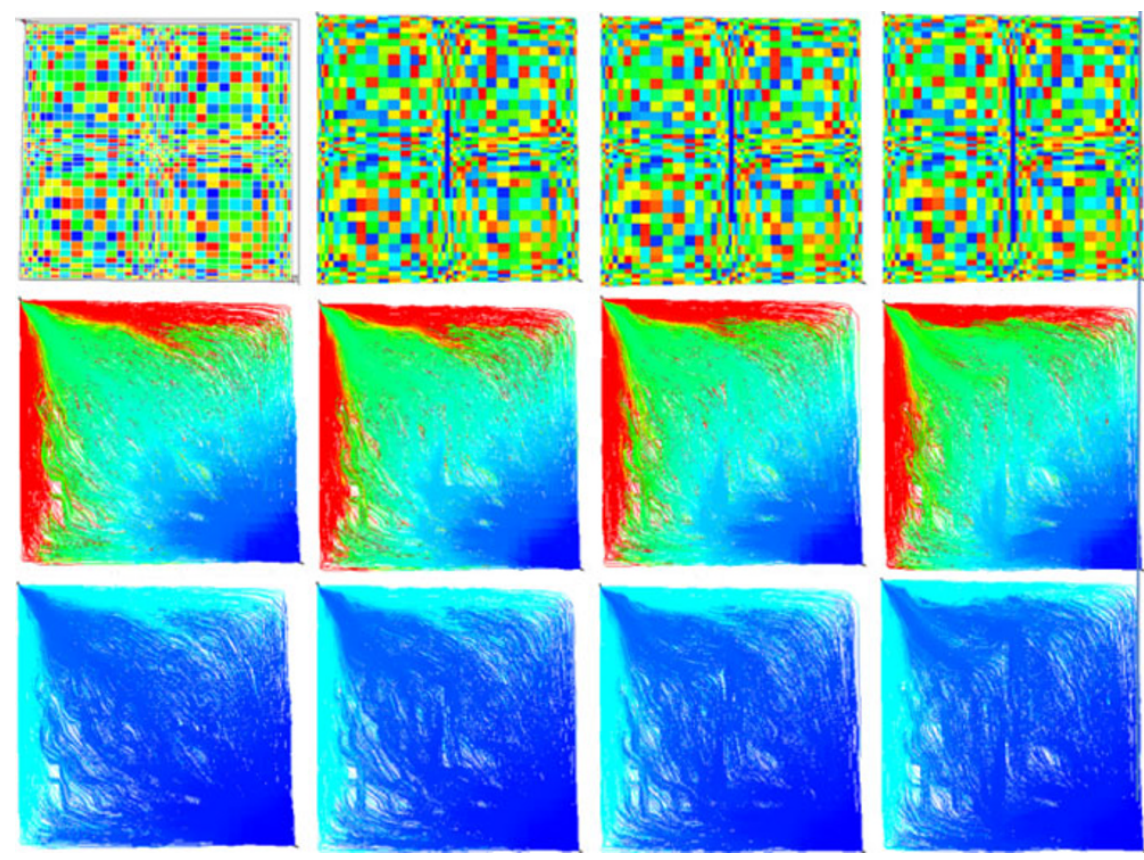

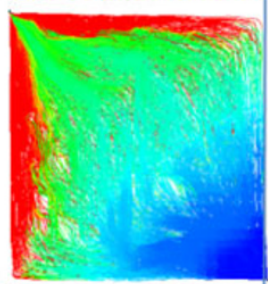

So, we believe it is fair to state that the application of barrier fracture to shutoff excess water production is not limited to specific reservoir type. As a matter of fact, Pirayesh et al. (2012) applied this innovative concept to a wide variety of conditions and various patterns of injection and proved its positive impact in improving sweep efficiency.

\section{Conclusions}

In this study, we created several streamline simulation models to show the power and validity of the innovative concept of barrier fracture. The streamline simulation models range from a simple homogeneous reservoir to a heterogeneous reservoir. The effect of different barrier fracture lengths on the reservoir performance was analyzed. We also built streamline models for conventional mechanical and chemical water shutoff techniques, recompletion and RPM, to compare them with the novel barrier fracture water shutoff technique. We conclude that:

- Based on the oil and water saturation distributions along streamlines of reservoirs with and without a barrier-fracture, a barrier-fracture has the ability to modify flow profile and divert the displacing fluid.

- Barrier-fractures can help to improve recovery by delaying water-breakthrough and improving the volumetric sweep efficiency of a water-flooding project.

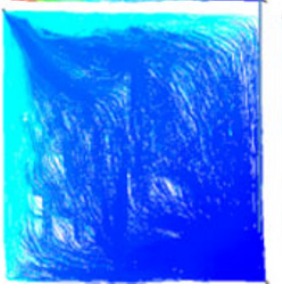

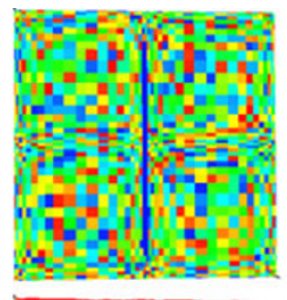
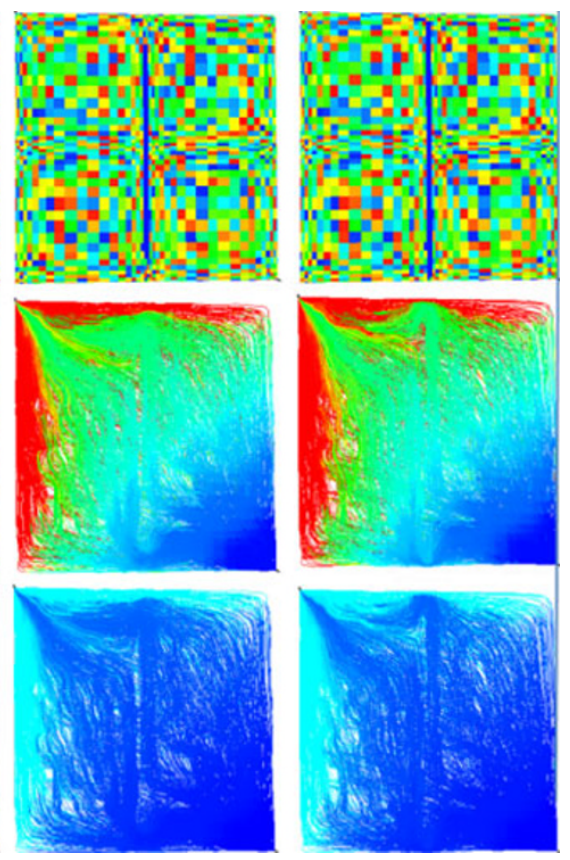

Fig. 19 Snapshots of oil and water saturations along the streamlines in the heterogeneous model at time 2,190 days 


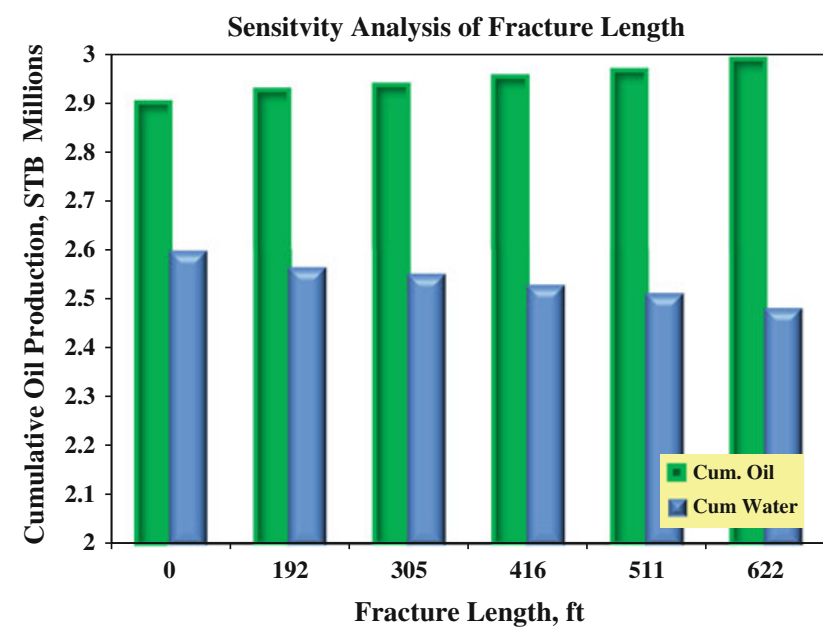

Fig. 20 Sensitivity analysis of fracture length showing increase in oil and decrease in water with increasing fracture length - Heterogeneous reservoir

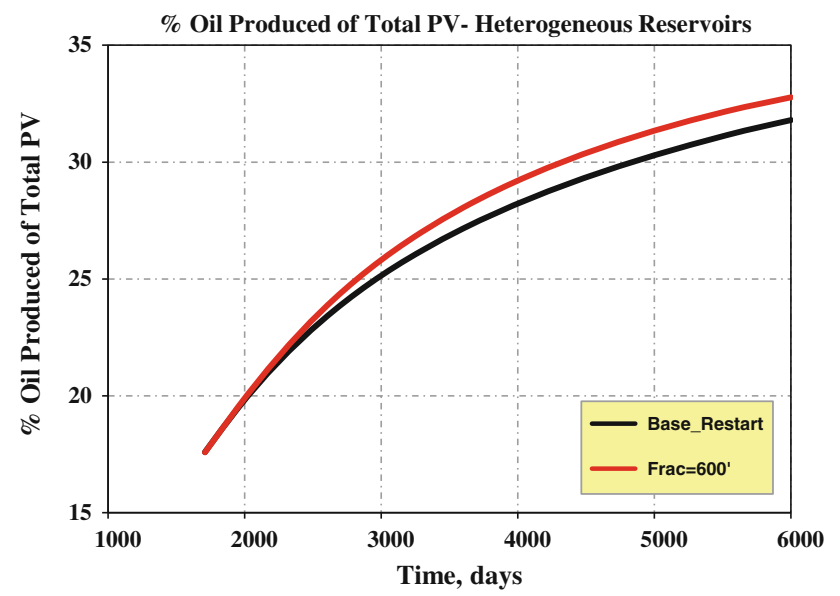

Fig. 21 Comparison of $\%$ oil produced of the base case and the $622^{\prime}$ barrier fracture length

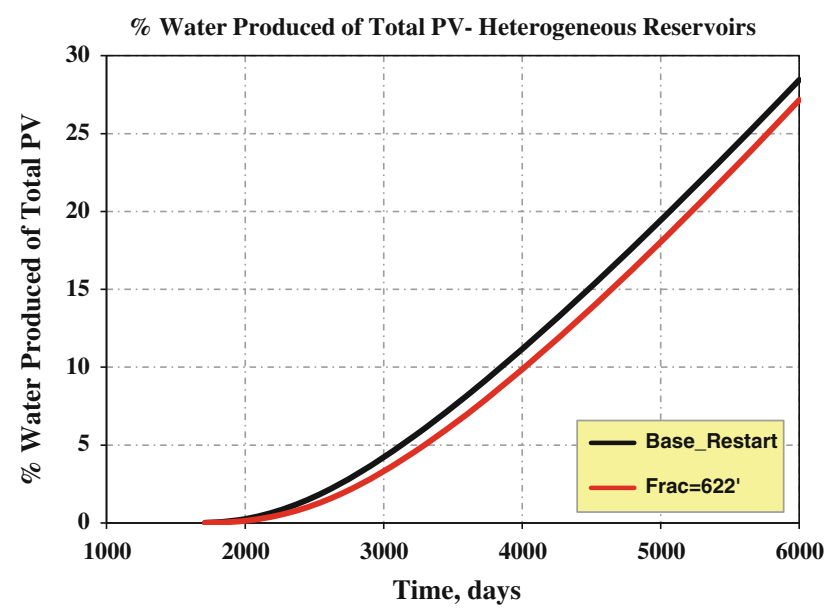

Fig. 22 Comparison of $\%$ water produced of the base case and $622^{\prime}$ barrier fracture length
- Oil production can be increased and water production decreased as a result of introducing a barrier-fracture into a reservoir that was under water-flooding.

- Models with longer barrier-fractures show better performance than those with shorter ones.

Open Access This article is distributed under the terms of the Creative Commons Attribution License which permits any use, distribution, and reproduction in any medium, provided the original author(s) and the source are credited.

\section{References}

Ansah J, Soliman MY, Ali S, Moreno C, Jorquera R, Warren J (2006) Optimization of conformance decisions using a new wellintervention simulator. SPE paper 99697 presented at the SPE/ DOE Symposium on Improved Oil Recovery, Tulsa, Oklahoma, USA, 22-26 April. doi:10.2118/99697-MS

Batycky RP (1997) A three dimensional two phase field scale streamline Simulation. PhD dissertation. Stanford University, Stanford, California (January 1997)

Benson I, Nghiem LX, Bryant SL, Sharma MM, Huh C (2007) Development and Use of a simulation model for mobility/ conformance control Using a $\mathrm{pH}$-sensitive polymer. SPE paper 109665 presented at the SPE annual technical conference and exhibition, Anaheim, California, U.S.A., 11-14 November. doi:10.2118/109665-MS

Dang Cuong TQ, Chen Z, Nguyen Ngoc TB, Bae W, Phung TH (2011) Development and optimization of polymer conformance control technology in mature reservoirs: laboratory experiments vs. field scale simulation. SPE paper 144221 presented at SPE enhanced oil recovery conference, Kuala Lumpur, Malaysia, 19-21 July. doi:10.2118/144221-MS

Cheng H, Oyerinde D, Datta-Gupta A, Milliken W (2006) Compressible streamlines and three-phase history matching. Paper SPE 99465 presented at the SPE/DOE Symposium on Improved Oil Recovery, Tulsa, Oklahoma, USA, 22-26 April. doi:10. 2118/99465-MS

Dalrymple ED, Creel P, Rohwer C, Crabb III H (1998) Results of Using a Relative-Permeability Modifier with a Fracture-Stimulation Treatment. SPE paper 49043 presented at the SPE Annual Technical Conference and Exhibition, New Orleans, Louisiana, USA, 27-30 September. doi:10.2118/49043-MS

Datta-Gupta A, King MK (2007) Streamline simulation: theory and practice. Textbook Series, SPE

Di Donato G, Huang W, Blunt M (2003) Streamline-based dual porosity simulation of fractured reservoirs. Paper SPE 84036 presented at the SPE annual technical conference and exhibition, Denver, Colorado, 5-8 October. doi:10.2118/84036-MS

Doi M, Suzuki S (2000) Formulation of the re-development scheme: reservoir modeling for the highly heterogeneous reservoir to assess IOR. Paper SPE 59440 presented at the SPE Asia Pacific conference on integrated modeling for asset management, Yokohama, Japan, 25-26 April. doi:10.2118/59440-MS

Garmeh R, Izadi M, Salehi M, Romero JL, Thomas CP, Manrique EJ (2011). Thermally active polymer to improve sweep efficiency of water floods: simulation and pilot design approaches. SPE paper 144234 presented at the SPE enhanced oil recovery conference, Kuala Lumpur, Malaysia, 19-21 July. doi:10.2118/ 144234-MS

Gautier Y, Nœtinger B, Roggero F (2001) History matching using a streamline-based approach and exact sensitivity coefficients. 
Paper SPE 71626 presented at the SPE annual technical conference and exhibition, New Orleans, Louisiana 30 September-3 October. doi:10.2118/71626-MS

Gerritsen MG (2008) Industrial compositional streamline simulation for efficient and accurate prediction of gas injection and WAG processes. Technical Report DE-FC26-06NT15530, United States Department of Energy, National Energy Technology Laboratory, Stanford, USA

Gerritsen MG, Jessen K, Mallison BT, Lambers J (2005) A fully adaptive streamline framework for the challenging simulation of Gas-Injection processes. Paper SPE 97270 presented at the SPE Annual Technical Conference and Exhibition, Dallas, Texas, 9-12 October. doi:10.2118/97270-MS

Hardy M, van Batenburg D (1997) Near-wellbore temperature simulations assist design of water-shutoff treatments. SPE paper 38196 presented at the SPE european formation damage conference, The Hague, Netherlands, 2-3 June. doi:10.2118/ 49043-MS

Herbas J, Moreno R, Romero MF, Coombe D, Serna A (2004) Gel performance simulations and laboratory/field studies to design water conformance treatments in Eastern Venezuelan HPHT Reservoirs. Paper SPE 89398 presented at the SPE/DOE Symposium on improved oil recovery, Tulsa, Oklahoma, USA, 17-21 April. doi:10.2118/89398-MS

Ibrahim MN, Clark RA Jr, Al-Matar BS (2007) Streamline simulation for reservoir management of a super giant: sabriyah field (North Kuwait) case study. Paper SPE 105069 presented at the SPE middle east oil and gas show and conference, Kingdom of Bahrain, 11-14 March. doi:10.2118/105069-MS

Ingebrigtsen L, Bratvedt F, Berge J (1999) A streamline based approach to solution of three-phase flow. Paper SPE 51904 presented at the SPE reservoir simulation symposium, Houston, Texas, 14-17 February. doi:10.2118/51904-MS

Jessen K, Orr Jr FM (2002) Compositional streamline simulation. Paper SPE 77379 presented at the SPE annual technical conference and exhibition, San Antonio, Texas, 29 September2 October. doi:10.2118/77379-MS

Lolomari T, Bratvedt K, Crane M, Milliken WJ, Tyrie JJ (2000) The use of streamline simulation in reservoir management: methodology and case studies. Paper SPE 63157 presented at the SPE annual technical conference and exhibition, Dallas, Texas, 1-4 October. doi:10.2118/63157-MS
Mallison BT, Gerritsen MG, Matringe SF (2006) Improved mappings for streamline-based simulation. SPE J 11(3): 294-302. Paper SPE 89352-PA. doi:10.2118/89352-PA

Melo R C B D, Aboud RS (2008) Over 100 conformance fracturing operations in Brazil: results and improvements. SPE paper 113624 presented at the Europec/EAGE conference and exhibition, Rome, Italy, 9-12 June. doi:10.2118/113624-MS

Moreno J, Kazemi H, Gilman JR (2004) Streamline simulation of countercurrent water-oil and gas-oil flow in naturally fractured dual-porosity reservoirs. Paper SPE 89880 presented at the SPE annual technical conference and exhibition, Houston, Texas, 26-29 September. doi:10.2118/89880-MS

Pirayesh E, Soliman MY, Rafiee M (2012) Improving horizontal and vertical sweep efficiencies in enhanced oil recovery techniques using fracturing. ACS National Meeting and Expo, Philadelphia, PA, 19-23 Aug

Thiele MR (2001) Streamline simulation. 6th international forum on reservoir simulation. Schloss Fuschl, Austria, 3-7 September 2001

Thiele MR, Batycky RP, Fenwick DH (2010) Streamline simulation for modern reservoir-engineering workflows. J. Pet Tech 62(1): 64-70. SPE 118608. doi:10.2118/118608-MS

Thornton K, Soliman MY, Jorquera R (2010) Optimization of InflowControl devices or mechanical conformance decisions using a new coupled well-intervention simulator. SPE paper 139435 presented at the SPE Latin American and Caribbean Petroleum Engineering Conference, Lima, Peru, 1-3 December. doi:10. 2118/139435-MS

Tobenna Okeke T, Lane R (2012) Simulation and economic screening of improved-conformance oil recovery by polymer flooding and a thermally activated deep diverting gel. SPE paper 153740 presented at the SPE western regional meeting, Bakersfield, California, USA, 21-23 March. doi:10.2118/144234-MS

Tsau JS, Hill AD, Sepehrnoori K U (1985) Modeling of permeability reducing vertical conformance treatments. SPE paper 13569 presented at the SPE oilfield and geothermal chemistry symposium, Phoenix, Arizona, 9-11 March. doi:10.2118/13569-MS

Vasquez J, Miranda C (2010) Profile modification in injection wells through relative permeability modifiers: laboratory validation and reservoir simulation. SPE paper 135107 presented at the SPE production and operations conference and exhibition, Tunis, Tunisia, 8-10 June. doi:10.2118/135107-MS 